\title{
Marketing électoral et données numériques personnelles aux Etats-Unis et en France: échanges interculturels de techniques, particularités juridiques et analyse politico-culturelle
}

\section{Electoral marketing and personal digital data in the United- States and France: intercultural exchanges of techniques, particularities of law and politico-cultural analysis}

\author{
BÉATRICE SOMMIER \\ Université de Bretagne Occidentale \\ beatrice.sommier@brest-bs.com
}

\begin{abstract}
Electoral marketing and personal digital data in the United-States and France: intercultural exchanges of techniques, particularities of law and politico-cultural analysis This article aims to understand the intercultural exchanges from the United States to France in terms of digital electoral marketing in the context of the American presidential elections from 2008 to 2016 and the French elections of 2012 and 2017. Based on documentary research to identify the American situation and on a qualitative survey carried out during the French presidential election of 2017, it shows the existence of technical similarities between the two countries. However, due to a different legal framework in the United States and France, practices of digital electoral marketing diverge. Then the article tries to understand the origin of these disparities between the two countries by mobilizing the Cultural Theory of the anthropologist Mary Douglas: it analyses how the French economic and political actors and the State seek to nego-
\end{abstract}

\begin{abstract}
Resumen
Esta investigación tiene como objetivo comprender los intercambios interculturales entre Estados-Unidos y Francia en relación con el marketing electoral numérico. Se interesa en las elecciones presidenciales estadounidenses entre 2008 y 2016 y francesas de 2012 y 2017. El artículo se basa en un análisis bibliográfico para identificar la situación estadounidense y en una encuesta cualitativa realizada durante las elecciones francesas de 2017 que permiten mostrar las semejanzas técnicas entre los dos países. Sin embargo, debido a un marco jurídico distinto entre Estados-Unidos y Francia, las prácticas de marketing electoral numérico no son las mismas. Este trabajo intenta explicar estas diferencias entre los dos países apoyándose en la Teoría Cultural de la antropóloga Mary Douglas. Acaba por analizar cómo en Francia los actores económicos, políticos y el Estado negocian hasta llegar a una situación donde las diferencias interculturales con Estados-Unidos disminuyen.
\end{abstract}


tiate together and attenuate intercultural gaps with the American situation.

\section{Key-words}

electoral marketing, personal digital data, intercultural exchanges between France and the United States, national rights, Cultural Theory.

\section{Palabras clave}

marketing electoral, datos numéricos personales, intercambios interculturales, Francia y Estados-Unidos, derechos nacionales, Teoría Cultural.

\section{Introduction}

Les Etats-Unis ont souvent été un objet de réflexion pour la France en matière politique, il n'est qu'à se souvenir du travail d'analyse de Tocqueville sur la démocratie en Amérique dont il vantait la liberté politique tel un remède efficace "pour combattre les maux que l'égalité peut produire" comme le despotisme (1981: 135). Depuis, des passerelles ont continué de se tisser entre les Etats-Unis et la France, en particulier en marketing électoral, aboutissant à une relation, plutôt à sens unique: la vie politique des Etats-Unis devenant non plus seulement un objet de réflexion mais un modèle pour la France. On considère habituellement que les techniques de marketing politique ont été mises en œuvre pour la première fois en 1952 lors de l'élection présidentielle américaine, avant de traverser l'Atlantique et d'être appliquées en France pour l'élection présidentielle au suffrage universel direct en 1965, autrement dit "avec un retard certain sur les Etats-Unis" (Maarek, 1992: 19). Plus spécifiquement, Albouy souligne que la campagne d'Eisenhower, s'est caractérisée par la volonté de "faire précéder les décisions stratégiques d'études préalables destinées à mieux connaître le 'marché' électoral pour mieux l'influencer" (1994: 9). Ces enquêtes ont permis de construire le discours politique tels le slogan directeur de campagne, les messages à diffuser dans des spots publicitaires devenus nombreux dans les médias de masse et centrés sur la personnalité du candidat. Ces nouvelles techniques d'études et de communication ont, dès lors, supposé l'introduction de professionnels du marketing dans les équipes de campagne. En premier lieu, les conseillers en communication, les spin doctors, dont le rôle est " $d$ 'influencer et de transformer (to spin) les idées ou les comportements des citoyens sans qu'ils en aient conscience" (Ollivier-Yanis, 2010: 31), en travaillant notamment sur le langage politique dont le sens provient à la fois “de l'interaction simultanée de différents phénomènes linguistiques et d'une situation de communication particulière" (Delmas, 2012: 104).

Ces techniques de marketing politique d'origine américaine se sont implantées dans les autres démocraties à partir des années 1960 “avec, il est vrai, un certain nombre d'adaptations liées aux particularités locales” (Albouy, 1994: 10). En France, en 1965, Lecanuet a recouru à ces méthodes, conseillé par Bongrand un professionnel de la communication politique ayant au préalable observé le marketing électoral aux Etats-Unis. Le fait qu'Eisenhower ait remporté l'élection étatsunienne et que Lecanuet ait obtenu un score plus élevé 
que prévu jusqu'à mettre de Gaulle en ballotage, a fortement contribué à faire reconnaître l'efficacité de ces techniques de marketing électoral. Elles se sont alors généralisées sur la période 1960-1970 aux Etats-Unis et 1970-1980 en France.

Quelques années plus tard, les Français ont continué de s'inspirer des Etats-Unis en analysant comment l'équipe d'Obama a utilisé les big data pour favoriser sa victoire en 2008, puis en 2012, à la fois par la mobilisation des citoyens et le ciblage personnalisé des électeurs permis par l'adaptation des canaux de communication et des éléments de langage. Le recours aux données numériques personnelles a alors fait son apparition en France: "En 2012, la campagne pour l'élection présidentielle française s'est ainsi fortement appuyée sur des bases de données d'électeurs" (Theviot, 2016: 139). Ce phénomène a pris une large ampleur en 2017 de sorte que les data "politiques ne sont plus une spécificité américaine, le numérique fait désormais pleinement partie des obligatoires de campagnes nationales hexagonales" (Theviot \& Treille, 2019: 72).

Cela dit, si la France s'inspire des Etats-Unis en matière d'utilisation électorale des data personnelles, il est intéressant de se demander si c'est une application trait à trait des techniques étatsuniennes ou si, comme l'indiquait Albouy au sujet des années 1960, ces emprunts supposent toujours des adaptations liées aux particularités locales. Quelles sont alors ces particularités locales susceptibles de créer des différences dans le recours aux données numériques personnelles lors des campagnes présidentielles entre les Etats-Unis et la France? Quelles adaptations engendrent-elles de la part des acteurs du champ politique et comment procèdent-ils? Parmi les acteurs du champ politique, outre les candidats et les professionnels de la communication, nous intègrerons l'Etat en ce qu'il définit un cadre règlementaire dans lequel évolue le marketing électoral (en revanche, les citoyens-électeurs n'apparaîtront qu'en filigrane dans cet article). Nous nous demanderons comment interagissent et communiquent ces trois acteurs et quel rôle jouent leurs discours dans ces adaptations locales. Précisons que nous nous intéresserons peu à la dynamique discursive elle-même (analyse internaliste du langage), mais davantage aux "contextes de production des langages [...] aux conditions sociopolitiques de leur réception" (Hamzaoui et al., 2019: 64) parmi ces trois acteurs. Notre approche sera donc plutôt externaliste. L'objet de cet article est donc de savoir si les échanges interculturels de techniques de marketing électoral supposent des adaptations locales et de cerner le rôle des discours d'acteurs du champ politique dans ces adaptations. A notre connaissance, il s'agit d'un objet de recherche non exploré à ce jour. Notre période d'analyse sera les campagnes présidentielles de 2008 à 2016 aux Etats-Unis et celles de 2012 et 2017 en France. Le choix de porter notre attention sur les élections présidentielles repose sur le fait que dans les deux pays, il s'agit de "l'évènement politique le plus mobilisateur" (Tiberj, 2004: 265), autrement dit, le contexte est favorable à la production d'interactions et de discours susceptibles d'influencer les acteurs retenus.

Pour ce faire, nous avons mis en œuvre une recherche documentaire et une enquête 
qualitative que nous commencerons par présenter avant de répondre à notre problématique en analysant trois dimensions du champ du marketing électoral concernées par ces échanges interculturels: les dimensions technico-économique, juridique et politico-culturelle.

\section{Méthodologie}

Afin de comprendre en profondeur le recours aux données numériques personnelles dans la campagne présidentielle française de 2017, d'identifier le rôle du marketing électoral américain dans cette utilisation, les interactions et les adaptations des acteurs français, nous avons conduit une recherche documentaire, réalisé des entretiens semi-directifs et de l'observation participante. La recherche documentaire avait notamment pour objectifs de cerner les pratiques électorales américaines en matière de big data et le cadre réglementaire dans lequel elles s'inscrivent. De même, elle visait à connaître l'arsenal législatif français en matière de recours aux données numériques personnelles dans le champ électoral. Douze entretiens d'une durée moyenne de $1 \mathrm{~h} 35$ ont été conduits pendant ou juste après la campagne présidentielle 2017. L'un auprès de la CNIL pour approfondir nos connaissances sur le plan légal, sept auprès de différentes équipes de campagne, trois auprès d'entreprises de solutions et/ou stratégie numériques électorales et un avec une spécialiste en analyse de données numériques pour appréhender les pratiques des acteurs (Annexe, tableau 1). Un guide d'entretien spécifique a été élaboré pour chacune de ces cibles. Enfin, l'observation participante visait à compléter une part de l'information reçue en entretien: nous avons été formés à une solution de stratégie électorale pour en saisir plus précisément les modalités de fonctionnement. La retranscription intégrale des entretiens et les notes prises pendant la formation au logiciel représentent un matériau de 351 pages. Nous avons retenu comme "unité d'enregistrement" le thème (Bardin, 1977: 135-138). Une lecture détaillée de l'intégralité de notre matériau a permis d'identifier soixante-et-onze thèmes. Des relations existant entre ceux-ci, nous avons réalisé un second niveau de codage en opérant des regroupements pour établir treize "catégories thématiques" (Bardin, 1977: 150-151).

\section{Résultats}

Nos résultats montrent entre les Etats-Unis et la France une continuité sur le plan technico-économique, mais une discontinuité sur le plan juridique que nous allons tenter d'expliquer et dont nous verrons les conséquences dans une troisième partie.

En premier lieu, sur le plan technico-économique, on observe des similitudes entre les outils de marketing électoral numérique existant aux Etats-Unis et ceux importés en France. Néanmoins, comme nous le verrons en seconde partie, sur le plan règlementaire, le cadre entre ces deux Etats-nations diffère, de sorte que les outils de collecte et d'utilisation des données numériques personnelles ne peuvent pas être appliqués strictement de la même 
manière entre les deux rives de l'Atlantique. Nous tenterons alors de comprendre dans un troisième temps d'où proviennent ces différences entre les deux pays grâce à l'anthropologie et à la Théorie Culturelle de Douglas, avant d'analyser comment les acteurs économiques et politiques français et la puissance publique cherchent malgré tout à composer pour tenter d'aboutir à une situation atténuant les écarts interculturels avec la situation étatsunienne.

\subsection{Continuité interculturelle sur le plan technico-économique}

Au niveau technico-économique, on constate l'existence d'une situation à la fois "d'interculturel factuel" et "d'interculturel volontaire" (Demorgon, 2003: 22-23), la campagne présidentielle française s'inspirant grandement des techniques de marketing électoral numérique développées Outre-Atlantique.

Les équipes de campagne interrogées nous ont permis de constater que quasiment tous les grands candidats à la présidentielle 2017 ont utilisé le logiciel NationBuilder, une plateforme américaine que l'on peut qualifier de " $C R M{ }^{1}$ appliqué aux questions politiques" selon le co-dirigeant d'une entreprise de solutions et conseil en stratégie numérique électorale. En effet, cette plateforme permet d'une part, de collecter des noms et des informations sur les sympathisants d'un.e candidat.e dans une base de données, de les mobiliser jusqu'à les transformer en militants, que ce soit par des dons en argent ou en temps et, d'autre part, de gérer les actions de communication et le contenu des messages de manière ciblée en fonction des informations détenues sur ces électeurs dans la base de données (caractéristiques sociodémographiques, centres d'intérêt, compétences, etc.). Le dirigeant d'une entreprise de conseil en stratégie numérique électorale sous-entendait plutôt une apparition factuelle que volontaire:
Personne n'avait vu arriver, et c'est le point majeur de la révolution de la data sur 2016- 2017 en France, NationBuilder. Et, l'arrivée de Nationbuilder a tout changé là-dessus parce que c'est un outil extrêmement puissant, très efficace [...] Ça a été utilisé par Mé- lenchon, $[\ldots]$ ça a été utilisé dans la primaire à droite par Juppé, Sarkozy, Fillon et Le Maire. [...] Donc, sur les gros candidats, à part Benoît Hamon, tout le monde a utilisé NationBuilder. Et, à En marche, Macron n'a pas utilisé NationBuilder [...] Mais dans la plateforme qui était à disposition des candidats aux législatives d'En marche, dedans, il y avait NationBuilder.

Cela dit, ce logiciel a pu être introduit volontairement dans certaines équipes de campagne par des personnes formées à son fonctionnement directement aux Etats-Unis:

1 CRM: Customer Relationship Management, logiciel permettant de générer de grandes bases de données pour, à l'origine, gérer la relation avec les clients dans les entreprises, ici avec les électeurs dans les équipes de campagne. Le logiciel auquel nous avons été formés part généralement de la liste électorale et des informations qu'elle contient (sexe, date et lieu de naissance, adresse), que les acteurs politiques complètent ensuite des informations que veulent bien leur donner les électeurs (e-mails, numéro de téléphone, centres d'intérêt...). 
C'était aussi une des raisons explicites de mon recrutement, c'est qu'est-ce qui se fait aux États-Unis... qu'on pourrait importer et adapter en France. Je m'étais auto-formée et donc je m'étais mise à mon compte pour déployer un logiciel qui s'appelle NationBuilder. (Responsable campagne numérique)

L'influence du marketing électoral étatsunien de la data est souvent passée par l'intermédiaire de jeunes gens ayant été militants ou simples observateurs des campagnes électorales américaines de 2008 à 2014, en particulier les présidentielles d'Obama en 2008 ou 2012 (Théviot, 2019). Fort de leur expérience et des compétences acquises, certains se sont ensuite fait salarier dans des équipes de campagne en France en 2017:

Quand, je suis parti aux États-Unis, j'ai fait cette campagne en 2014 [...] donc, eux, utilisaient des outils vraiment très, très performants qui étaient développés par le parti démocrate [...] Et, les Républicains, pareil, ils utilisaient le soutien en interne. Et c'était, en fait, des énormes machines tout-en-un qui... on insérait les données et on pouvait faire des appels automatiques, on pouvait faire du porte-à-porte, tout était organisé, du mapping électoral. (Responsable campagne numérique); Et j'ai travaillé en stratégie digitale pour... et des clients américains et français à mon compte après un passage dans la campagne de Barack Obama en tant que militante. (Responsable campagne numérique)

D'autres ont créé en France leur propre logiciel de traitement des données numériques inspiré des techniques mises en œuvre par l'équipe d'Obama:

On finissait nos études, et c'est là on a découvert les campagnes à la 'Obama', pour aller rapidement, avec un peu le cocktail qui nous plait toujours aujourd'hui qui est l'alliance de la technologie, la scientificité avec le contact humain et le porte-à-porte. [...] On avait des profs d'Harvard qui bossaient pour la campagne d'Obama. (Co-dirigeant d'une entreprise de solutions et conseil en stratégie numérique électorale)

Ces recrutements et créations d'entreprise concrétisent certaines recommandations émises dans un rapport publié par le laboratoire d'idées Terra Nova en janvier 2009 au titre explicite Moderniser la vie politique: innovations américaines, leçons pour la France. Ce rapport a analysé la campagne d'Obama de 2008 en réalisant " 80 entretiens exclusifs et approfondis avec les principaux acteurs de la campagne à Washington, New York et Chicago en novembre et décembre 2008" (Terra Nova, 2009: 5). Dans sa "leçon n 4", ce rapport souligne qu'Obama a fait des nouvelles technologies l'épine dorsale de sa campagne avec notamment la plateforme $M y B O$ (créée par Chris Hughes un des fondateurs de Facebook) à la fois comme outil de communication et de mobilisation pour les opérations de terrain (dont le porte-à-porte) et les dons. Dans sa "leçon n ${ }^{\circ} 5$ ", le recours aux bases de données est qualifié de "rupture orwellienne": outre la base de données constituée de toutes les informations recueillies pendant la campagne et intégrées dans $M y B O$, Obama a acheté la base Catalist qui contenait jusque six cents informations sur cent quarante millions d'Américains inscrits 
sur les listes électorales. Les croisements entre ces bases de données, mais aussi avec des sondages, a permis de modéliser les comportements des électeurs pour faire du micro-targeting, un ciblage personnalisé pour mieux convaincre les électeurs de voter Obama. En conséquence, dès 2009, ce rapport recommandait pour la France "d'investir dans une épine dorsale numérique" (Terra Nova, 2009: 76-78) et de constituer des bases de données et des modélisations permettant de cibler finement les électeurs. Il précisait également les obstacles juridiques, financiers et culturels à une application termes à termes en France des solutions observées aux Etats-Unis, nous y reviendrons. D'autres sources ont pu inspirer les Français, comme cet article d'Issenberg publié le 19 décembre 2012 dans la MIT Technology Review, intitulé "How Obama's Team Used Big Data to Rally Voters". Ce texte indique entre autres qu'en 2012, l'équipe de campagne -constituée de cinquante-quatre personnes rien que dans le département d'analyse de données- savait identifier les soixante-dix millions d'Américains ayant voté Obama en 2008 et avait mis en place des outils pour tester les messages de communication et éléments de langage les plus efficaces sur les médias les plus fréquentés par les électeurs potentiels d'Obama. Pène ajoute que la diffusion des pratiques de data électorales s'est aussi faite:

à travers des échanges entre experts, lors de conférences - comme celle organisée par le Personal Democracy Forum à Paris fin 2012-, [...] ou via des agences organisant des 'tours' de benchmark permettant aux professionnels d'identifier les meilleurs techniques. (2013: 137)

En France, les techniques étatsuniennes de marketing électoral numérique ont été suivies partiellement en 2012: mise en œuvre avec succès du porte-à-porte 2.0 pour le PS $^{2}$, création de plateformes similaires à $M y B O$ appelée Coopol pour le PS et Créateurs de possibles pour l'UMP, mais bientôt abandonnées. Il faut préciser que ces techniques font alors “l'objet de dénigrement" (Théviot \& Treille, 2019: 75). Ce n'est véritablement que pour la présidentielle de 2017 que l'on peut parler de généralisation et de légitimation de ces techniques chez une majorité des candidats:

Les réactions que j'avais il y a deux ans et les réactions qu'on a maintenant après la victoire d'Emmanuel Macron sont pas du tout les mêmes. Donc je pense que maintenant la nécessité en est comprise. (Responsable campagne numérique)

On retrouve ce discours a fortiori du côté des entreprises qui ont intérêt à vendre leur solution au plus grand nombre de politiques:

2 Une des entreprises de stratégie numérique électorale que nous avons interrogée a défini les zones où les militants PS pouvaient effectuer le porte-à-porte, ce grâce à un logiciel croisant les résultats aux élections depuis 2002 par bureau de vote et les renseignements INSEE du recensement. Il s'est agi alors de déterminer les îlots où les militants avaient le plus de probabilités de convaincre des électeurs de voter Hollande en fonction des votes antérieurs dans ces îlots et des caractéristiques sociodémographiques. 
Quand on a commencé, il y a quatre ou cinq ans, c'était vraiment les types les plus intéressés qui faisaient ça. Aujourd'hui, si vous n'avez pas ce genre d'outil, vous commencez un peu à être un ringard

précise un co-dirigeant d'une entreprise de solutions en stratégie numérique ayant accompagné l'équipe d'Hollande en 2012 avant d'élargir son portefeuille de clients en 2017. Une adéquation a eu lieu entre les équipes de campagne et les entreprises de logiciel de stratégie électorale dont le nombre a progressé “à partir de 2014” (Theviot, 2018: 44):

L'offre a créé la demande. Le fait qu'on soit là, le fait qu'il y ait des éditeurs français qui s'en emparent, ça a créé effectivement la demande. Et un taux d'adoption qui est grandissant. (Dirigeant d'une entreprise de solutions en stratégie numérique)

Cependant, comme nous l'ont certifié certains interlocuteurs, le recours aux données numériques en France en 2017 est moindre qu'aux Etats-Unis:

Quand on parle d'analyse big data en fait on n'évolue pas du tout au même niveau quand on parle de réels big data électoral aux États-Unis qu'en France [...] Je ne sais pas si on peut parler d'un retard. Il y a peu de gens à être qualifiés, mais en tout cas, c'est assez incomparable en réalité. (Responsable campagne numérique)

Ce décalage en France provient notamment de ce que les équipes de campagne ont peu recruté de professionnels de la data:

Ce n'est pas rationalisé, c'est très désorganisé, y a pas de... ce qu'il faut c'est des data scientists. Les gens qui... dont c'est le métier [...] Il faut des spécialistes du marketing politique et ça, n'y en a pas. (Responsable campagne numérique)

L'équipe d'un député aux États-Unis, ou d'un congress man, c'est entre quinze et vingt personnes dans son bureau déjà! En France, c'est deux ou trois. (Dirigeant d'une entreprise de solutions en stratégie numérique)

Mais il est d'autres raisons à cette moindre diffusion en France des data à des fins électorales que nous expliciterons ultérieurement.

A l'analyse de ces discours d'informateurs, on comprend donc qu'il y a en marketing électoral numérique une influence des Etats-Unis sur la France, souvent factuelle mais aussi revendiquée et volontaire chez certains acteurs français, aboutissant à une continuité interculturelle sur le plan technico-économique. En ce sens, le développement du recours aux data est une nouvelle étape dans ce que Farrell, Kolodny \& Medvic qualifiaient déjà en 2001 d"'Américanisation des processus de campagne électorale" dans les démocraties occidentales (2001: 25). Est-il pour autant possible de parler de culture globale en matière de marketing électoral numérique? Ce serait oublier les différences que l'on voit poindre entre les deux pays. Nous allons à présent nous demander si elles proviennent des obstacles 
juridiques, financiers et culturels propres à la France que le rapport de Terra Nova pointait en 2009.

\subsection{Particularités sur le plan juridique}

Les élections présidentielles ont la particularité de se dérouler dans le cadre d'un Etat-nation, soit, d'être limitées aux frontières d'une nation que Sieyès définissait comme une collectivité d'individus vivant sous la même loi (Braud \& Burdeau, 1983). Les EtatsUnis et la France étant deux Etats-nations différents, ils disposent chacun d'un arsenal juridique propre, reposant sur une philosophie distincte. Le modèle américain est: "fondé sur le pragmatisme, l'optimisme et l'autorégulation; c'est la confiance dans la convergence des intérêts individuels et l'intervention de l'État a lieu seulement en cas de déséquilibre excessif" (Tijardovic, 2003: 187). Dans cette logique, les acteurs politiques, et économiques sont peu contraints par l'Etat et ils ont intérêt à faire converger leurs objectifs avec les droits des citoyens. Car ce modèle, qualifié de private enforcement prévoit que le citoyen dispose de moyens judiciaires efficaces qu'il peut saisir pour se défendre contre des violations de ses droits. En termes de données numériques personnelles en marketing électoral, cela se traduit par une plus grande liberté laissée aux acteurs politiques et entreprises de stratégie numérique pour collecter, stocker et utiliser des data personnelles sans obtenir le consentement préalable des électeurs concernés y compris des informations relatives à la religion, à l'appartenance ethnique et politique des citoyens, les électeurs ayant la possibilité de se saisir de la justice américaine en cas d'abus.

Concernant la législation relative au financement de la vie politique, en janvier 2010, via son arrêt 'Citizens United', la Cour Suprême des Etats-Unis a autorisé les entreprises et les syndicats à financer les campagnes électorales en versant des fonds sans limite à des $\mathrm{Su}$ per PAC (Super Political Action Committees) qui soutiennent un candidat ou un parti à condition qu'il n'y ait pas de lien direct entre ces donateurs et l'équipe de campagne officielle des candidats. Cette décision a eu pour conséquence, dès la campagne présidentielle de 2012, de faire qu'Obama et Romney ont renoncé aux fonds publics (qui auraient limité leurs dépenses à 84 millions de dollars) pour opter pour ce mode de financement privé sans borne. En 2014, la Cour Suprême a d'une certaine manière renchéri sa décision concernant les personnes morales, en déplafonnant cette fois les dons de particuliers. Elle a ainsi ouvert les vannes à un large financement privé et sans limite des campagnes électorales.

En France, le cadre législatif est plus contraignant pour les acteurs politiques et économiques, mais il est plus protecteur pour les citoyens. Il existe une règlementation visant à protéger les données personnelles des citoyens et le respect de celles-ci est assuré par l'autorité publique. Il s'agit d'un système de public enforcement puisque l'Etat ou l'autorité publique à qui il délègue ce rôle, la CNIL, agit pour prévenir, dissuader et sanctionner les 
comportements illégaux des entreprises ou acteurs politiques par divers moyens (auditions, informations, enquêtes, avertissement, amendes, etc...).

Il y a d'abord, effectivement, un rôle en amont des opérations de communication lancées par les candidats et les partis, qui est d'informer les candidats et de les accompagner dans leur mise en conformité [...] Et même pendant les périodes électorales à proprement parler, de campagne électorale officielle, nous répondons encore, bien entendu, dès que nous sommes contactés. Et puis au niveau des signalements, ceci permet aussi des ajustements en cours de campagne. (Responsable CNIL intervenant sur les questions institutionnelles et parlementaires)

Plus spécifiquement, la loi du 6 janvier 1978 indique que les données personnelles ne peuvent être collectées et utilisées par un tiers sans le consentement libre, spécifique, éclairé et univoque de la personne. En outre, elle précise en son article 6: "Il est interdit de traiter des données à caractère personnel qui révèlent la prétendue origine raciale ou l'origine ethnique, les opinions politiques, les convictions religieuses".

Or face à certaines nouvelles technologies des réseaux sociaux comme les API (Application Programming Interface) qui permettent la transmission de données personnelles d'une entité à une autre de manière automatisée, le risque que les citoyens ne puissent donner leur consentement est grand. En effet, la spécialiste en analyse de données numériques que nous avons interrogée expliquait:

L'API, y'a pas d'interface, de chaîne humain-machine, mais simplement une adresse internet, à laquelle le programme va accéder, et l'API vient avec son adresse -souvent des clés d'authentification, des clés spéciales développeurs-, qui va après contrôler et les programmes informatiques vont avoir le droit de faire un certain nombre de choses. Donc l'API vient avec un protocole d'interactions. Sur Twitter, par exemple, on va pouvoir aller heu... chercher le profil de la personne. Ça va être son nom sur Twitter. Ça va être la p'tite ligne qu'il a rentrée manuellement pour se décrire. Ça va être éventuellement sa géolocalisation, s'il l'a renseignée. Et puis, ça va être la liste de ses followers et des gens que lui-même suit.

Un dirigeant d'une entreprise de conseil en stratégie numérique électorale explicitait ce qu'il est techniquement possible de faire en politique grâce aux $A P I$ :

Le problème, c'est les $A P I$, les réseaux sociaux. [...] toutes les données que les gens mettent publiquement sur les réseaux sociaux donnent énormément d'indications sur eux, sur leurs centres d'intérêt, sur leurs goûts. Il y a d'abord [...] des gens qui réagissent devant un débat télé, donc des gens qui s'expriment publiquement sur des sujets politiques, quelque part, ils dévoilent leur opinion politique ou en tout cas leur inclinaison à un moment ou un autre. A partir du moment où ils le font publiquement, c'est pas anormal qu'on puisse s'en saisir 'tiens, j'ai vu que vous aviez publié ça, ça nous intéresse, est-ce que vous voudriez en faire plus à nos côtés?' [...] Et, ces choses-là sont, aujourd'hui, revendues par les réseaux sociaux pour faire du ciblage publicitaire, 
mais elles sont aussi accessibles à ceux qui veulent faire un ciblage en termes de communication électorale.

Pour chercher à protéger les citoyens de ces nouveaux risques, la Loi pour une République numérique du 7 octobre 2016 est venue compléter celle de 1978 en affirmant que "toute personne dispose du droit de décider et de contrôler les usages qui sont faits des données à caractère personnel la concernant". Il s'agit d'une affirmation du droit qu'ont les citoyens de disposer librement de leurs données ${ }^{3}$.

Cela dit, il n'existe pas en France de loi spécifique concernant l'utilisation des données numériques personnelles des électeurs par les acteurs politiques ou les entreprises de solutions numériques électorales. Et face à l'augmentation du recours à cette utilisation lors des présidentielles de 2012 et plus encore de 2017, la CNIL a dû réagir afin d'adapter le cadre légal aux évolutions technologiques et aux pratiques des acteurs du champ politique. Elle a ainsi publié une délibération le 26 janvier 2012 portant une recommandation relative à la mise en œuvre, par les partis politiques ou candidats, de fichiers dans le cadre de leurs activités politiques ${ }^{4}$. De même, le 8 novembre 2016, la CNIL a publié sur son site web un guide intitulé Communication politique: quelles sont les règles pour l'utilisation des données issues des réseaux sociaux $?^{5}$, précisant sa doctrine quant à la collecte de données des réseaux sociaux en vue d'actions de communication politique. Pour élaborer cette doctrine, la CNIL a auditionné les principales entreprises de solutions numériques électorales dès le premier semestre $2015^{6}$.

Pour ce qui est du financement de la vie politique en France, il repose d'abord sur la Loi du 11 mars 1988 qui a fixé les bases d'un financement par l'Etat de la vie politique (les comptes de campagne doivent être publiés, en échange l'Etat rembourse une partie des frais de campagne) et prévoyait un plafonnement des dépenses de campagnes nationales et des dons des personnes physiques et morales. Puis la Loi du 19 janvier 1995 a assuré la séparation du monde économique et politique en matière de financement, les entreprises ne pouvant désormais plus financer des partis ou des candidats. Les recettes des candidats proviennent donc uniquement d'apports personnels, de contributions de leur parti et de dons de

3 Bien qu'il n'était pas en vigueur pour la présidentielle 2017 qui nous intéresse dans cet article, il faudrait ajouter depuis mai 2018, le Règlement Général sur la Protection des Données venu renforcer la protection des citoyens puisqu'il rend nécessaire d'informer au préalable l'intéressé de la finalité de la collecte de ses données, impose un accord "matérialisé", qui exclut la possibilité d'un accord verbal et donne la possibilité à la personne dont les données sont collectées de retirer son consentement à tout moment.

4 Cette délibération est accessible par le biais du site internet Légifrance: $<$ https://www.legifrance.gouv.fr/affichCnil.do?oldAction=rechExpCnil\&id=CNILTEXT000025364626\&fastReqId $=1082859898 \&$ fastPos $=1>$ [18/02/2021].

$5<$ https://www.cnil.fr/fr/communication-politique-quelles-sont-les-regles-pour-lutilisation-des-donnees-issues-des-reseaux $>[18 / 02 / 2021]$.

6 Conférence de presse de présentation du 37 $7^{\mathrm{eme}}$ rapport d'activité 2016 et des enjeux 2017 du 27 mars 2017 de la CNIL. <https://www.cnil.fr/sites/default/files/atoms/files/dossier_de_presse_cnil_bilan_2016_et_enjeux_2017. $\operatorname{pdf}>[18 / 02 / 2021]$. 
personnes physiques qui, depuis la Loi du 17 mai 2013, ne peuvent dépasser $4600 €$ par an et par personne physique. La législation française est donc opposée à celle des Etats-Unis: le financement est plafonné et l'Etat rembourse près de $50 \%$ des dépenses engagées dès lors que le candidat a obtenu au moins $5 \%$ des suffrages exprimés au premier tour.

En réalité, ce cadre réglementaire différent entre les Etats-Unis et la France engendre des pratiques distinctes des acteurs par-delà les possibilités de continuité technologique. Certains interlocuteurs rencontrés précisent que les électeurs français connaissent leurs droits et savent qu'ils peuvent saisir la CNIL en cas de communication politique trop dense:

Les gens savent, en France, qu'il y a une loi informatique et libertés. Ils savent qu'on peut saisir la CNIL [...] Les citoyens français n'accepteront pas de se retrouver demain matin sous un flot continu de messages politiques, ça ne marchera pas, il y aura un rejet. (Dirigeant d'une entreprise de solutions et/ou conseil en stratégie numérique électorale)

Ceux ayant une expérience américaine soulignent d'autant mieux le fait que l'obligation française du consentement individuel limite la collecte de données personnelles et partant les actions de marketing électoral personnalisé au profit d'actions par îlots:

En France, on ne peut pas accumuler légalement ces types de données sans avoir à chaque fois eu un opt-in enfin un go explicite... Ce modèle d'analyse big data où justement on peut croiser tous types de sets de données qui permettent d'identifier des profils, mais qui correspondent à des individus. Ce modèle-là n'existe pas dans les stratégies électorales françaises parce qu'il est illégal. [...] En France, ce que vous... ce qu'on a le droit de faire, c'est de prendre même à l'échelle du quartier ou du lot, donc un endroit de 2000 personnes définies par l'INSEE ou du bureau de vote. (Responsable campagne numérique)

Cette particularité du droit français explique que la fonction 'Match Contact' du logiciel américain NationBuilder a dû être déprogrammée en France. Cette fonction permet, à partir de l'adresse e-mail donnée par un électeur à un candidat, de capter via des API de l'information laissée par ce même électeur sur Facebook, LinkedIn, Twitter et Meetup (localisation, biographie du compte, 'likes', 'retweets' et 'follows') afin d'incrémenter la base de données de ce candidat et l'aider à personnaliser ses messages de communication. Or si l'électeur a volontairement laissé son e-mail, il n'a toutefois pas consenti au captage de ses données sur les réseaux sociaux. La CNIL a précisé ce point dans son guide du 8 novembre 2016 alors que les équipes étaient déjà en campagne et "NationBuilder a dit qu'il suspendait le système de matching automatique" (Dirigeant d'entreprise de conseil en stratégie numérique électorale). Ajoutons que nos interlocuteurs connaissent l'interdiction de ficher certaines informations personnelles délicates en France, mais courantes aux Etats-Unis: 
Le ciblage racial et religieux, ça ne marche pas. Aux États-Unis, ça fait un carton parce que c'est la manière dont ils conçoivent le marketing politique. [...] Donc eux, ils vont trouver ça génial de cibler la communauté noire baptiste de l'église de tel quartier, qui correspond à telle communauté du bureau de vote... Ils savent qu'il y a tant de personnes à la messe le dimanche. Eux, ils trouvent ça génial. Mais chez nous, ce n'est pas possible. Et ça ne sera jamais le cas. (Dirigeant d'une entreprise de solution numérique électorale)

La plus grande protection des données numériques personnelles en France comparée aux Etats-Unis dissuade peut-être aussi davantage les abus. Un dirigeant d'une entreprise de conseil en stratégie numérique électorale l'exprimait ainsi:

De fait, il n'y a pas eu d'excès en France, il n'y a pas eu de Cambridge Analytica en France [...] Sans doute grâce à la CNIL qui a quand même mis une vraie pression sur les partis politiques. (Dirigeant d'une entreprise de conseil en stratégie numérique électorale)

Dans notre enquête, certains interlocuteurs ont tout de même mentionné l'affaire de l'application knockin. Cette application, conçue dans une équipe de campagne, se basait sur les données personnelles capturées grâce à la fonction 'Match Contact' de NationBuilder pour identifier les personnes à cibler prioritairement en porte-à-porte. La CNIL a lancé une enquête au sujet de cette application suite à une médiatisation du sujet, pour finir par conclure en février 2017, que l'application en soi satisfaisait aux exigences de la Loi informatique et libertés. Tout autre a été la portée de l'affaire Cambridge Analytica partie des Etats-Unis puis devenue scandale international: fin mars 2018, la presse a révélé, et Marc Zuckerberg a confirmé, que 87 millions d'utilisateurs de Facebook dans le monde (dont un peu plus de 200000 Français) ont vu leurs données personnelles transmises à leur insu à Cambridge Analytica. Cette société britannique a analysé des données numériques personnelles pour cerner les comportements, mais aussi la personnalité des électeurs en vue d'influencer leur vote, notamment pendant la présidentielle américaine de 2016 en diffusant sur leur compte des publicités ciblées en faveur de candidats conservateurs ou nationalistes, tel Trump dans certains Etats américains (Huchon, 2018). Cela dit, si un décalage important existe à ce jour entre la France et les Etats-Unis dans le recours aux data pour des raisons juridiques, on peut se demander avec Theviot jusque quand la CNIL pourra servir de rempart face à ce pouvoir inhérent à la mobilisation des big data en politique: "En France, la CNIL exerce encore une protection forte, mais les enjeux économiques amènent à s'interroger sur la vente de données personnelles" (2018: 50). Notre dernière partie apportera des éléments de réponse.

Enfin, les divergences en matière de financement politique expliquent les écarts colossaux entre les budgets de campagne aux Etats-Unis et en France. A titre indicatif, en 2008, pour la présidentielle aux Etats-Unis, Obama a dépensé 712 millions de dollars et MacCain 326 millions (Terra Nova, 2009: 60), quand en France pour les élections de 2007, les dé- 
penses officielles de Sarkozy au second tour s'élevaient à 20,9 millions d'euros ${ }^{7}$ et à 20,6 millions pour Royal ${ }^{8}$, le plafond des dépenses autorisés par la loi en France étant toujours situé à 22 millions d'euros depuis. De là découle notamment la différence de moyens techniques, mais surtout humains en matière de data dont faisait état l'interviewé cité plus haut qui mentionnait quinze à vingt personnes dédiées aux data dans une équipe de campagne aux législatives américaines, contre seulement deux ou trois en France et qu'un responsable de plateforme numérique exprimait en ces termes:

En réalité, on s'est très vite trouvés confrontés à un problème que j'avais signalé dès le début, mais on ne m'a pas écouté: c'est qu'on peut avoir des fichiers fantastiques avec des niveaux de détails excellents, mais s'il n'y a personne pour envoyer des mails et pour penser le niveau de détail quand on est tout en bas de l'arbre disons, vous arrivez à rien. Donc voilà, comme on a de tout petits moyens et qu'on n'est pas beaucoup, et bien on n'est pas en capacité de faire des propositions d'actions qui entrent dans ce niveau de détails-là.

$\mathrm{Au}$ fond, les particularités sur le plan juridique de chacun des deux pays donnent lieu à des différences dans les pratiques de data électorales qu'un interviewé résumait pour la France en trois limites: l'interdiction de recueillir certaines informations personnelles, le faible nombre des experts et de l'offre techniques, ainsi que le manque de formation des équipes de campagne à l'utilisation des données:

1) La première, c'est la limite de la data, parce qu'on ne les a pas toutes pour une raison légale. C'est bête, mais avoir la religion de quelqu'un, dans l'absolu, ça éclaire énormément sur son vote. Mais ça on l'aura jamais. Et c'est tant mieux, mais en tout cas, on l'aura pas quoi. Donc y a la limite de la data qui manque de richesse [...]. 2) Y a le problème du traducteur de la data, y a encore peu de data scientists, en France, y a peu de logiciels, donc y a pas de concurrence énorme, y a pas d'améliorations énormes, c'est ce problème-là et 3 ) le troisième problème qui est l'un des plus importants, c'est le problème des ressources humaines et puis de la formation des militants. Voilà, c'est la data, sa traduction et son utilisation. Ça, c'est les trois limites.

Ces divergences entre les Etats-Unis et la France expliquent donc le retard que certains interlocuteurs associaient au marketing électoral de la data en France.

A l'issue de ces deux premiers niveaux d'analyse, on constate donc l'existence d'une culture technico-économique commune aux deux pays, mais d'une culture juridique différente, la singularité culturelle des lois expliquant certaines différences de pratiques de marketing électoral numérique dans les deux pays, par-delà le recours aux mêmes techniques. Ce résultat nous conforte à considérer avec Verbunt (2012: 27-28) et contrairement au courant anthropologique américain Culture et Personnalité que Verbunt mobilise

7 Selon $<$ https://www.legifrance.gouv.fr/jorf/id/JORFTEXT000017847611> [19/04/2021].

8 Selon $<$ https://www.legifrance.gouv.fr/jorf/id/JORFTEXT000017847538> [19/04/2021]. 
à travers une citation de Benedict, qu'il n'est plus approprié d'“attribuer une culture à une société", mais de "préciser de quelle culture il s'agit" (technique, juridique...), les sociétés partageant entre elles à la fois certains champs culturels communs et certaines différences culturelles.

Il nous reste à présent à nous demander d'où proviennent ces différences quant à l'arsenal juridique étatsunien d'une part et français d'autre part (ce private vs public enforcement), et à nous interroger pour savoir si elles sont indépassables comme le sous-entend le dernier interlocuteur cité. Pour ce faire, nous nous appuierons sur l'anthropologie à la fois culturelle et structuraliste de Douglas.

\subsection{Des différences culturelles entre les Etats-Unis et la France, à leur atténuation par la négociation dans le marketing électoral numérique en France}

La philosophie sous-jacente aux deux systèmes juridiques disparates entre la France et les Etats-Unis, autrement dit le public vs private enforcement, fait écho à deux des quatre modèles d'organisation sociale opposés que Douglas développe dans sa Théorie Culturelle. Le premier est le "type hiérarchique": "Il s'agit d'un système de coordination fondé sur l'autorité, les pratiques antérieures, la règle [...]. Le mode hiérarchique suppose l'autorité fondée sur une gestion avisée." (Douglas, 2007: 490). Dans ce modèle d'organisation sociale collectiviste, l'Etat et la bureaucratie ont une place importante et le contrôle de l'information est strict dans le but de défendre l'intérêt du plus grand nombre. Le second modèle d'organisation sociale est le "type entrepreneurial ou individualiste", en opposition avec le précédent,

parce qu'il autorise explicitement la compétition individuelle. En tant que système de coordination, il repose sur la manifestation du pouvoir, soit par la coercition soit par la richesse. Il dispose d'un système propre d'incitations. (Douglas, 2007: 490)

Douglas ajoute que:

les contraintes de la bonne gestion passent au second plan par rapport à la promotion du commerce [...]. L'individualisme tendra à promouvoir des politiques de court-terme, plus opportunistes et méritocratiques, au nom de la liberté et de la prospérité. (Douglas, 2007: 492 $)^{9}$

Autrement dit, la liberté d'action et d'initiative est encouragée dans cette culture.

On retrouve dans l'organisation sociale de type entrepreneurial le modèle culturel des Etats-Unis et particulièrement de son système juridique de type private enforcement.

9 Les deux autres modèles culturels développés par Douglas sont la culture 'sectaire' émergeant d'un groupe contestataire aux principes égalitaires et communautaires et la culture des 'isolés' caractérisée par l'apathie et le fatalisme de personnes dominées. 
En effet, l'importance donnée aux individus, à la méritocratie (la figure du self-mademan), les valeurs de liberté économique et politique bien soulignées par Tocqueville (1981) et l'idée selon laquelle la somme des intérêts particuliers constitue l'intérêt général, engendrent une moindre présence de l'Etat et de son appareil juridique, les personnes physiques (ici les citoyens) et morales (en l'occurrence, les entreprises de solutions numériques électorales) étant censées s'autoréguler, ces dernières cherchant à faire prospérer leur commerce. A l'inverse, l'organisation sociale hiérarchique ressemble davantage au modèle culturel français de l'Etat-Providence construit de la fin du XIXème siècle au sortir de la Seconde Guerre Mondiale et au cadre juridique de type public enforcement: la loi protège les citoyens, plus faibles que les personnes morales, en octroyant une moindre liberté aux acteurs politiques et économiques. De même, un organisme étatique comme la CNIL représente l'autorité et est garante du respect des règles par les acteurs politiques et les entreprises.

Ces deux modèles d'organisation sociale sont présentés comme opposés par Douglas elle-même et certains auteurs lui ont reproché -outre le fait d'envisager les cultures comme antagonistes-, la rigidité de sa théorie culturelle plus propice à penser la réalité sociale au niveau "méso et macro" qu'au niveau des individus (Girard, 2013: 140).

Comme nous nous sommes situés depuis le début de cet article au niveau macrosocial en cherchant à comparer les Etats-Unis et la France, la théorie de Douglas apparaît pertinente pour interpréter nos résultats. Mais elle peut nous servir tout autant pour ajouter le niveau méso-social à la suite de notre analyse, en nous situant uniquement en France cette fois, dans le but de comparer les objectifs des acteurs économiques, politiques et étatique (la CNIL). Douglas souligne que ces tendances culturelles "ne forment pas des cultures séparées" (2007: 489), qu'elles peuvent donc co-exister au sein d'un même pays. Elle précise même: "Dans une démocratie accomplie, les quatre types de coordination ainsi que leurs tendances culturelles fondatrices s'affrontent avec vivacité" (Douglas, 2007: 492-493). C'est particulièrement le cas entre la culture hiérarchique et la culture individualiste: "Il existe par exemple une "diagonale positive" qui relie les partisans d'un ordre hiérarchique et les entrepreneurs" (Douglas, 2007: 495). Les groupes individualistes peuvent alors apporter des initiatives nouvelles de l'extérieur. Selon nous, il en est ainsi des jeunes Français qui, formés aux outils de stratégie numérique électorale aux Etats-Unis, les ont ensuite importés dans les équipes de campagne de la présidentielle française. S'ils se sont d'abord heurtés à des résistances en 2012, ils ont été accueillis favorablement en 2017, car le recours aux données numériques personnelles a montré son efficacité dans les campagnes électorales aux Etats-Unis et les acteurs politiques français ont eu le temps d'intégrer l'idée d'un "pouvoir des algorithmes" (Theviot \& Treille, 2019: 73). Il en est de même de ces entrepreneurs venus questionner la culture hiérarchique en proposant des outils ou du conseil en stratégie numérique électorale dont le nombre et l'offre se sont 
développés sur cette même période créant leur propre demande, comme nous l'avons vu. Reste la CNIL qui, en conformité avec sa culture hiérarchique, a continué de faire respecter les lois et a renforcé la règle de protection des données personnelles des électeurs via certaines publications et des enquêtes en cas de soupçon de manquement au cadre juridique de la part d'acteurs politiques et économiques de culture individualiste.

Pourtant dans ce contexte présidentiel aux enjeux colossaux, nous observons que loin de s'être montrées antagonistes, la culture hiérarchique de la CNIL et la culture individualiste des entreprises de stratégie numérique électorale et des équipes de campagne (en particulier leur responsable numérique) ont pu échanger et négocier pour parvenir à certains accords susceptibles de donner satisfaction aux représentants des deux types d'organisation sociale. L'analyse de ces échanges nous invite alors à nous intéresser au rôle qu'ont joué les discours de ces acteurs sur leur capacité à s'influencer et à s'adapter mutuellement. En l'occurrence, leurs interactions discursives ont suivi deux sens: logiquement dans notre culture française principalement hiérarchique, on les observe de la CNIL vers les autres acteurs, mais notre recherche montre aussi qu'elles ont eu lieu des entreprises vers cette instance étatique. Ainsi, d'une part, en amont de la campagne présidentielle 2017, rappelons que la CNIL a auditionné et écouté les arguments des entreprises de solutions numériques pour comprendre les évolutions technologiques en marketing électoral et adapter la rédaction de ses guides:

Nous avons amorcé une réflexion au premier semestre 2015 qui s'est poursuivie jusqu'au début de l'année 2016 avec les principaux concepteurs de logiciels de prospection politique. Voilà, donc nous les avons auditionnés. (Responsable intervenant sur les questions institutionnelles et parlementaires de la CNIL)

Ensuite, la CNIL a rencontré les équipes de campagne pour les informer des évolutions règlementaires et les contraindre à respecter la loi notamment en recourant à l'argument suivant très bien retenu par un dirigeant d'entreprise de conseil en stratégie numérique électorale interrogé: "Cent pour cent des électeurs dont vous avez piqué les données ne voteront pas pour vous". D'autre part, nos entretiens auprès d'entreprises montrent qu'il y a eu des échanges discursifs soutenus sous forme de lobbying exercé par ces dernières à l'encontre de la CNIL pour assouplir certaines règles du fait notamment d'un contexte de primaires nombreuses:

La CNIL, en 2012, quand il y a eu la première primaire qui était la primaire socialiste, avait exigé que les fichiers soient détruits après la primaire. Là [2016-2017], on a - je dis 'on' parce qu'on était plusieurs à faire du lobbying pour nos différents clients - on a fait un lobbying assez intense auprès de la CNIL pour qu'elle change sa vision des choses. Certes, il y avait cinq primaires, donc ça changeait un peu la donne, qui était de dire 'des gens qui viennent à la primaire, il est légitime qu'on en garde le contact et qu'on puisse continuer à s'adresser à eux' [...]. La CNIL a donc changé sa manière de 
faire les choses cette fois-ci. (Dirigeant d'une entreprise de conseil en stratégie numérique électorale)

En réalité, sur ce point précis, la position de la CNIL est un peu plus nuancée comme en témoigne sa doctrine: les fichiers contenant les contacts occasionnels doivent être détruits à la fin des opérations électorales. Seul le "fichier des sympathisants", constitué des personnes ayant consenti à devenir "contacts réguliers" du parti, peut être conservé à l'issue des élections primaires. Il n'en demeure pas moins que certaines entreprises ont essayé par ailleurs de faire pression sur la CNIL pour qu'elle autorise l'usage de la fonction 'Match Contact' de NationBuilder. Cette fois, le lobbying relatif à l'autorisation d'utiliser cette fonction américaine de captage de data personnelles a échoué, démontrant que la CNIL exerce toujours son rôle de rempart face au recours aux données numériques personnelles. Nonobstant, à travers ces deux exemples de discours de pression exercé par les entreprises de solution numérique électorale, on conçoit aussi que les enjeux économiques et politiques sont tels dans une élection présidentielle, qu'on peut se demander jusque quand l'Etat tiendra. Car les acteurs économiques et leurs clients politiques convaincus par les data ${ }^{10}$ savent aussi mobiliser l'argument de l'intérêt général ou de l'aide à la puissance publique, prouvant ainsi leur capacité à adapter leurs "stratégies communicatives dans l'interaction avec autrui" (Delmas, 2010: 111) en endossant des éléments de langage traditionnellement énoncés par l'Etat. Ainsi lors de ma formation au logiciel de stratégie électorale, la possibilité offerte par cette solution de cibler les abstentionnistes pour les inciter à aller voter a été citée à deux reprises. De même, cette idée a été exprimée en entretien:

Dans ce contexte-là, on a quand même vu, pour certains clients, ça permet quand même de mobiliser leur électorat... Il y a un vrai usage, je ne veux pas dire d'intérêt public, mais bon quand même... Ça contribue à faire voter quand même un petit peu... Ne serait-ce que d'informer qu'il y a une élection. (Co-dirigeant d'une entreprise de solutions et conseil en stratégie numérique électorale)

Du côté des acteurs politiques, une responsable de campagne numérique est allée jusqu'à souligner dans son discours que l'utilisation de logiciel comme NationBuilder facilite en réalité le travail de contrôle de la CNIL:

10 Cette précision sur la conviction des acteurs politiques s'impose, car même si tous reconnaissent l'importance désormais prise et l'efficacité des data dans les campagnes électorales, tous ne sont pas prêts à exercer du lobbying pour développer leur utilisation. Certains sont même critiques vis-à-vis des data car ils en connaissent les travers comme dans le cas du scandale de Cambridge Analytica auquel fait allusion un secrétaire général: "c'est ce qui est en train d'être creusé en termes d'investigations journalistiques sur des pratiques de Donald Trump [...] vous voyez bien qu'il y a cette crainte de... la manipulation électorale par l'utilisation des big data". En réalité, plus les personnes dans les équipes de campagne sont impliquées dans le recours aux data, plus elles sont prêtes à intervenir auprès de la puissance publique pour défendre leur extension en marketing politique. 
[...] ça constitue une énorme amélioration dans la protection et le suivi des données des particuliers. Il faut se rappeler qu'avant...l'émergence et la généralisation d'outils du type NationBuilder ou autre qui permettent ne serait-ce qu'à la CNIL de pouvoir avoir un lieu, centralisé, contrôlable avec un historique sur chacune des fiches de comment elle avait été traitée, et caetera et d'où elle venait, par opposition à l'avant.

Autrement dit, on observe des interactions et des pressions contraires, inhérentes à la divergence de culture et d'intérêt entre les acteurs de culture individualiste et celui d'organisation hiérarchique: les équipes de campagne et leurs prestataires numériques cherchant à qualifier au maximum des bases de données de citoyens à des fins de communication et de mobilisation électorale, tandis que la CNIL aspire à limiter cette qualification pour protéger les données numériques personnelles de ces mêmes citoyens. Cette instance étatique exerce alors sa mission de coordination par la voie de l'autorité sur les autres parties, tandis que pour leur part, les entreprises et les politiques expriment leur pouvoir via l'incitation et le lobbying, au nom de la prospérité et de la liberté. Il en résulte un travail collaboratif basé sur des échanges discursifs et des négociations entre les différentes parties prenantes qui permet d'aboutir parfois à des adaptations locales telle la possibilité désormais donnée aux acteurs politiques de conserver des fichiers d'électeurs aux primaires dès lors que ceux-ci ont consenti à devenir des contacts réguliers et pas seulement occasionnels. On se retrouve alors dans cette situation que décrivait Douglas:

s'il se produit un décalage entre la culture locale, une organisation locale [ici la réglementation française] et la manière dont le monde extérieur les affecte [l'influence des outils numériques de marketing électoral américain], l'organisation et la culture s'adapteront toutes les deux. (2007: 493)

Néanmoins, en s'appuyant sur les recherches du linguiste Nonhoff, on peut aussi se questionner pour savoir si les aspirations parfois antagonistes aujourd'hui entre, d'une part, les acteurs politiques et économiques et, de l'autre, la CNIL, n'aboutiront pas demain à un discours équivalent chez ces trois acteurs portant sur la nécessité d'un recours libéralisé aux données personnelles, à fortiori dans une élection aux enjeux tels qu'une présidentielle (exemple, conservation des fichiers de contacts occasionnels, autorisation de la collecte automatisée de données personnelles publiques issues des réseaux sociaux). En effet, en procédant par analogie, on peut se demander si les stratagèmes et les discours d'expertise et de lobbying des premiers finiront par devenir hégémoniques, à l'instar de "l'hégémonie de la formation discursive qui s'est constituée autour de la demande d'une 'économie sociale de marché' dans le discours économico-politique allemand” (Nonhoff, 2007: 78) des années 1940 aux années 1990. 


\section{Conclusion}

Nous avons montré l'existence d'échanges interculturels des Etats-Unis vers la France, aboutissant à l'utilisation d'outils de $C R M$ et au recrutement de spécialistes des data dans les équipes de campagne à la présidentielle française, particulièrement en 2017, dans l'esprit des campagnes d'Obama. Ces échanges technico-économiques n'engendrent pourtant pas des pratiques de marketing électoral numérique strictement communes entre les deux pays du fait d'un arsenal juridique différent. La France cherchant davantage à protéger les données numériques personnelles des citoyens par l'intervention de l'Etat (consentement obligatoire, interdiction de ficher l'appartenance ethnique, religieuse ou politique des individus), les acteurs politiques et économiques français sont plus contraints dans leur pratique de marketing numérique personnalisé. En somme, la singularité des lois dans ces deux Etats, reposant elle-même sur deux cultures différentes, plutôt hiérarchique pour la France et individualiste pour les Etats-Unis, explique les différences de pratiques de marketing électoral numérique, par-delà le recours aux mêmes techniques.

Pour autant, tout comme Douglas précise que les cultures hiérarchiques et individualistes peuvent s'affronter dans une même société, notre recherche montre aussi qu'en France, les acteurs économiques et leurs clients politiques porteurs de la culture individualiste ne se contentent pas de respecter le droit existant en matière de recours aux données numériques personnelles en marketing politique, ils ont intérêt à négocier et influencer la culture hiérarchique incarnée par la CNIL pour faire évoluer le cadre juridique national dans un sens plus favorable à leurs pratiques, gage alors d'une plus grande prospérité. Ils s'appuient alors sur des échanges discursifs (auditions, lobbying) dont l'intensité est inhérente au contexte de la présidentielle et de ses enjeux et qui engendrent des adaptations des techniques de marketing électoral étatsuniennes aux particularités culturelles et juridiques françaises.

Cela dit, cette recherche s'arrêtant à l'élection présidentielle française de 2017, il serait intéressant de suivre l'évolution de ces trois acteurs, de leurs interactions et de leurs discours lors de la campagne de 2022 et de cerner leurs antagonismes, leurs adaptations, de voir aussi si une stratégie hégémonique offensive s'opère en faveur d'un recours aux données numériques personnelles moins contraint à l'image de ce qui se fait aux Etats-Unis. 
Anales de Filología Francesa, n. ${ }^{\circ}$ 29, 2021

BÉATRICE SOMMIER

\section{Références bibliographiques}

Albouy, Serge. 1994. Marketing et communication politique. Paris, L'Harmattan.

BARdin, Laurence. 1977. L'analyse de contenu. Paris, PUF.

Braud, Philippe \& François Burdeau. 1983. Histoire des idées politiques depuis la Révolution. Paris, Editions Montchrestien.

Delmas, Virginie. 2012. "Pour une analyse pluridimensionnelle du discours: le discours politique" in La linguistique, vol. 48, 103-122.

Demorgon, Jacques. 2003. “L'interculturel entre réception et invention. Contextes, médias, concepts" in Questions de communication, $\mathrm{n}^{\circ} 4,43-70$.

Douglas, Mary. 2007. "Pour ne plus entendre parler de la "culture traditionnelle"” in Revue du MAUSS, $\mathrm{n}^{\circ} 29,479-516$.

Farell, David M., Robin Kolodny \& Stephen Medvic. 2001. "Parties and Campaign Professionals in a Digital Age. Political Consultants in the United States and their Counterparts Overseas", in Press/Politics, n 6 (4), 11-30.

Girard, Tobias. 2013. “Comment pense Mary Douglas? Risque, culture et pouvoir” in Ethnologie française, vol. 43, 137-145.

Hamzaoui, Ouassim, Tania Jimenez, Christèle Lagier \& Eric SanJuan-Ibekwe. 2019. "Contextualisation du discours politique" in Document numérique, vol. 22, 63-84.

Huchon, Thomas. 2018. "Fake America Great Again. Comment on a manipulé l'Amérique" in Documentaire, 2DF/Arte.

IssenberG, Sasha. 2012. "How President Obama's campaign used big data to rally individual voters" in MIT Technology Review, 19 décembre, 156-194.

MaArex, Philippe J.1992. Communication et marketing de l'homme politique. Paris, Litec.

NoNHOFF, Martin. 2007. “L'analyse hégémonique: à propos du développement récent de l'analyse de discours politique dans les sciences politiques" in Langage et société, n 120, 77-90.

Ollivier-Yaniv, Caroline. 2010. "Discours politiques, propagande, communication, manipulation" in Mots. Les langages du politique, $\mathrm{n}^{\circ}$ 94, 31-37.

Pene, Clémence. 2013. "La nouvelle 'science électorale' américaine” in Politique étrangère, n² été, 127-139.

Terra Nova. 2009. "Moderniser la vie politique: innovations américaines, leçons pour la France" in Rapport de la mission d'étude de Terra Nova sur les techniques de campagne américaines, janvier, 132-154.

Theviot, Anaïs. 2016. "Les data: nouveau trésor des partis politiques? Croyances, constitutions et usages comparés des données numériques au Parti Socialiste et à l'Union pour un Mouvement Populaire" in Politiques de communication, $\mathrm{n}^{\circ}$ 6, 137-166. 
Theviot, Anaïs. 2018. “Un nouveau marché politique du big data électoral?” in Communication \& Organisation, $\mathrm{n}^{\circ}$ 54, 41-53.

Theviot, Anaïs. \& Eric Treille. 2019. "Mobiliser par les données. Les stratégies numériques de campagne des candidats à la primaire de la droite et du centre de 2016 " in Politiques de communication, $\mathrm{n}^{\circ} 12,71-96$.

TiBerJ, Vincent. 2004. “ Compétence et repérage politiques en France et aux Etats-Unis: une contribution au modèle de 'l'électeur raisonnant" in Revue française de science politique, vol. 54, 261-287.

Tijardovic, Stéphane. 2003. "La protection juridique des données personnelles. Vers une nécessaire adaptation de la norme juridique aux évolutions du monde numérique" in Les Cahiers du numérique, vol. 4, 185-203.

Tocqueville, Alexis (de). 1981. De la démocratie en Amérique. Paris, Garnier-Flammarion.

Verbunt, Gilles. 2012. "Comment l'interculturel bouscule les cultures" in Les Cahiers Dynamiques, $\mathrm{n}^{\circ} 57,22-28$. 


\section{Annexe Tableau 1: caractéristiques de l'échantillon des entretiens}

\begin{tabular}{|c|c|c|}
\hline Entretien & $\begin{array}{c}\text { Type d'acteur dans le champ } \\
\text { politique }\end{array}$ & Fonction \\
\hline 1 & CNIL & $\begin{array}{l}\text { Responsable intervenant sur les ques- } \\
\text { tions institutionnelles et parlementaires }\end{array}$ \\
\hline 2 & Équipe de campagne & $\begin{array}{l}\text { Responsable communication numérique } \\
\text { (candidat de gauche) }\end{array}$ \\
\hline 3 & Équipe de campagne & $\begin{array}{l}\text { Responsable de la plateforme numé- } \\
\text { rique (candidat de gauche) }\end{array}$ \\
\hline 4 & Équipe de campagne & Secrétaire général (candidat de gauche) \\
\hline 5 & Équipe de campagne & $\begin{array}{l}\text { Directeur de campagne (candidat de } \\
\text { gauche) }\end{array}$ \\
\hline 6 & Équipe de campagne & $\begin{array}{l}\text { Responsable campagne numérique (can- } \\
\text { didat de droite) }\end{array}$ \\
\hline 7 & Équipe de campagne & $\begin{array}{l}\text { Responsable campagne numérique (can- } \\
\text { didat de droite) }\end{array}$ \\
\hline 8 & Équipe de campagne & Conseiller national (candidat de droite) \\
\hline 9 & $\begin{array}{l}\text { Entreprise de solutions et conseil en } \\
\text { stratégie numérique électorale }\end{array}$ & Co-fondateur et co-dirigeant \\
\hline 10 & $\begin{array}{l}\text { Entreprise de solutions et/ou conseil } \\
\text { en stratégie numérique électorale }\end{array}$ & Co-fondateur et dirigeant \\
\hline 11 & $\begin{array}{l}\text { Entreprise de solutions et/ou conseil } \\
\text { en stratégie numérique électorale }\end{array}$ & Fondateur et dirigeant \\
\hline 12 & $\begin{array}{l}\text { Experte en analyse de données nu- } \\
\text { mériques }\end{array}$ & $\begin{array}{l}\text { Enseignante-chercheure spécialiste de } \\
\text { l'analyse des réseaux sociaux et respon- } \\
\text { sable d'une formation de data-scientist }\end{array}$ \\
\hline
\end{tabular}


